\title{
ESPECIFICIDAD DE LA CLÍNICA PSICOANALÍTICA CON NIÑOS. CONSIDERACIONES DEL NIÑO COMO SUJETO DEL LENGUAJE
}

\section{THE SPECIFICITY OF PSYCHOANALYTIC PRACTICE WITH CHILDREN CONSIDERING CHILDREN AS A SUBJECT OF LANGUAGE}

\section{VIRNA PINOS Z. ${ }^{1}$}

Recibido: 15 de mayo de 2017

Aceptado: 29 de agosto de 2017

\footnotetext{
${ }^{1}$ Pontificia Universidad Católica del Ecuador, Facultad de Psicología, Quito, Ecuador (vpinos861@puce.edu.ec).
} 



\section{ESPECIFICIDAD DE LA CLÍNICA PSICOANALÎTICA CON NIÑOS. CONSIDERACIONES DEL NIÑO COMO SUJETO DEL LENGUAJE}

\section{THE SPECIFICITY OF PSYCHOANALYTIC PRACTICE WITH CHILDREN CONSIDERING CHILDREN AS A SUBJECT OF LANGUAGE}

VIRNA PINOS Z.

PALABRAS CLAVE: Sujeto del lenguaje, clínica psicoanalítica, síntoma, transferencia, cura.

KEY WORDS: Subject of language, psychoanalytic practice, symptom, transference, cure.

\section{RESUMEN}

El presente artículo constituye del lenguaje. Apunte necesario que consuna reflexión a partir de conceptos funtituye el eje de las intervenciones que el damentales que planteados desde el clínico se proponga y que marca el punto psicoanálisis lacaniano, permiten una delimitación del campo de la clínica con niños, cuya especificidad se sostiene en de quiebre de un campo que a lo largo de la historia ha generado debates y cuesla consideración del niño como sujeto tionamientos en la pretensión de ubicarlo haciendo parte de las psicoterapias. 


\begin{abstract}
This article involves a reflection supported over some fundamental concepts of the Lacanian psychoanalysis which allows a specific delimitation on the clinical practice with children. This specificity is based over the premise that the child is a subject of language. The assumption of the child as a subject of

language must be the main idea from which the analyst will interview in a psychoanalytical way. It marks a breaking point that throughout history has brought out a lot of debates and questioning because clinical practice with children has been taken only inside the psychotherapy area.
\end{abstract}

\title{
INTRODUCCIÓN
}

La clínica psicoanalítica con niños trabajada desde hace varios años por reconocidos psicoanalistas pertenecientes tanto a la escuela inglesa como a la escuela francesa del Psicoanálisis, ha planteado diversos modos de abordaje de los conflictos psíquicos del niño acordes a una conceptualización que, como en el caso de Anna Freud se apoyaban en una psicología del Yo, en articulación con aspectos educativos y pedagógicos como necesarios en la conducción terapéutica de los niños. De otro lado, concepciones desarrollistas como las propuestas por Karl Abraham que confundieron la estructuración subjetiva con una maduración progresiva ligada a la superación de estadios en pos de la constitución de un objeto sexual completo, fue otra de las vertientes que influyó en las intervenciones clínicas con niños; al igual que los aportes de Melanie Klein quien encontró como ejes de su práctica la búsqueda en los tiempos más precoces de la infancia, de las coordenadas de la estructuración simbólica en las posiciones: depresiva y esquizo-paranoide. Sin embargo, frente a estos planteamientos, se encuentran otros modos de comprensión de la subjetividad del niño, que toman en cuenta la singularidad de su constitución en los entretejidos del lenguaje, es decir otorgándole un lugar como sujeto del mismo, como es el caso de Francoise Doltó o Jean Bergès, siendo esta la línea conceptual que guiará la argumentación del presente artículo.

En efecto al hablar de clínica psicoanalítica con niños nos vemos abocados a un campo con características particulares, que obligan al clínico a mantenerse en una constante reflexión 
de su práctica. Conceptos como deman$\mathrm{da}$, deseo, transferencia, intervenciones analíticas, entre otros, están sujetos a una permanente interrogación en el trabajo con los niños, a partir de la consideración del lugar que ocupan en el Otro. Es decir en el tesoro de significantes que nos antecede y nos determina, así lo planteaba Jean Bergès al decir: "En el niño el Simbólico es primero", (Bergès, 2011, p.23) ya que desde el nacimiento o incluso antes de este, el infans se encuentra bañado de lenguaje y por lo tanto está sumergido en los deseos de ese Otro, que por esta vía se encarnan dándole una forma subjetiva, ... el niño está forzado al habla y al lenguaje; está atrapado en la cadena de lo que se dice; esto no es un baño de sonido, las conversaciones del entorno, se arman en las leyes de la articulación y de la sintaxis, es un forzamiento sostenido por las leyes del lenguaje". (Bergès, en Rey \& Bergès-Bounes, 2008, p. 93). Este es uno de los ejes fundamentales que sostiene una práctica psicoanalítica rigurosa y que apunta no a la consideración del niño como susceptible de acciones pedagógico - educativas, en la búsqueda de una integración adecuada a lo social, sino que apunta al sujeto que en su malestar, hace escuchar el deseo en el que se constituye.

Varios son los debates que persisten en la actualidad respecto a la clínica con niños, alguno de ellos hace referencia a quienes manifiestan que esta clínica es del orden de la psicoterapia, mientras otros como Doltó defienden incluso que el psicoanálisis casi puro, el verdadero psicoanálisis es aquel que se realiza con los niños. He ahí otro eje de discusión que la especificidad de esta clínica exige; sin embargo, el presente artículo no se centrará en esta polémica sino que intentará reflexionar sobre elementos propios al quehacer clínico que den cuenta de dicha especificidad'.

La relevancia de una reflexión de esta naturaleza guarda relación con los efectos a nivel clínico, que una determinada postura marca como hilo conductor de la así llamada cura, concepto cuyas implicaciones y determinaciones en el caso del trabajo con niños, también será abordado.

\footnotetext{
${ }^{1}$ Especificidad: cualidad y condición de específico. Adecuación de algo al fin al que se destina. (Recuperado de: http://dle.rae.es/?id=GWuT7Lq)
} 


\section{FUNDAMENTO TEÓRICO}

La complejidad y el campo especifico del psicoanálisis con niños, demandado casi siempre como opción psicoterapéutica para "aliviar" alguna angustia que las preguntas de los niños, vestidas de síntomas, despiertan en la escuela o en la familia, conmina al clínico a tener presente en su práctica, elementos cuya determinación conceptual mar- cará el pulso de las intervenciones que se proponga al recibir niños en su consulta, no sin olvidar que justo ahí donde el malestar aparece, hay un sujeto del inconsciente que se hace escuchar.

A continuación se abordarán algunos de los puntos más importantes que intentarán delinear la especificidad de esta clínica.

\section{EL SÎNTOMA EN EL NIÑO}

Para Freud el síntoma era la expresión de un conflicto inconsciente que al igual que los sueños y los actos fallidos estaba cargado de sentido. También catalogado como "formación de compromiso", el síntoma tiene por función el cumplimiento de deseo y la realización de un fantasma inconsciente que sirve al cumplimiento del mismo (Chemama, 2004, p.638). Es decir que el síntoma muestra algo del contenido inconsciente y al mismo tiempo lo reprime, escondiéndolo. Este es uno de los puntos más complejos en el trabajo con niños ya que la demanda de ayuda por parte de los padres, muchas veces no va a coincidir con lo que en el niño origina su sufrimiento.

En relación con este aspecto, en un pequeño texto escrito por Jacques
Lacan en 1969, bajo el título: "Nota sobre el Niño", encontramos importantes reflexiones a ser consideradas. Lacan nos hace saber la implicación que el síntoma del niño tiene, en primer lugar en tanto "respuesta a lo que hay de sintomático en la estructura familiar. El síntoma puede representar la verdad de la pareja en la familia", o en segundo lugar, el síntoma "involucrado directamente como correlativo de un fantasma", en ese sentido, el niño depende de la subjetividad y sobre todo del deseo de la madre, es decir, "deviene el objeto de la madre, y ya no tiene otra función que la de revelar la verdad de ese objeto. El niño realiza la presencia de lo que Jacques Lacan designa como objeto a en el fantasma" (Lacan, 2012, p.393).

En cuanto al síntoma del niño como respuesta a la verdad de la pare- 
ja conyugal, Lacan nos advierte que es quizá el campo más complejo, pero al mismo tiempo el más abierto a las intervenciones. Y es que la posibilidad de "responder" con su síntoma a los avatares del deseo, da cuenta de la constitución de un sujeto que aún "en ciernes", si se puede decir así, interroga la posición del Otro marcando así su falta. A este respecto Alba Flesler, psicoanalista que trabaja con niños, plantea la importancia de ubicar en el análisis lo que ella llama: "tiempos del sujeto", los cuales se articulan a los tiempos del Edipo planteados por Freud y conceptualizados por Lacan como tiempos lógicos en los que el sujeto se constituye. En cada uno de ellos el sujeto adviene de la articulación significante, en donde el intervalo permite la posibilidad de respuesta al deseo del Otro. Este movimiento se observa ya en el primer tiempo en el cual el niño pese al estado de prematuración, como lo llama Lacan, a nivel de la corporalidad aún indefenso y dependiente casi por completo de ese Otro que le asegura la supervivencia; sin embargo, "responde" con mirada, voz y postura corporal a la demanda que este le dirige, evidenciando así un movimiento de ida y vuelta que produce un intercambio. El espacio vacío que este movimiento suscita, hace posible una respuesta subjetiva en donde lo pulsional se manifiesta haciendo saber de la entrada del significante en el cuerpo. Un claro ejemplo de ello es la clínica con bebés, en donde permanentemente somos testigos de las primeras respuestas del sujeto al Ilamado del Otro generalmente parental.

"Las intervenciones regulares del Otro primordial durante los dos primeros meses de vida del bebé, apuntan a eliminar las situaciones de desamparo desesperantes para él, así como las fuentes de estimulación desorganizadora más fuertes. Ellas también van a permitir la realización de giros positivos de llamado y de respuesta cada vez más adecuada, sobre todo en relación a la alimentación y a los cuidados, el surgimiento del objeto real, ahí donde lo espera e imagina el bebé, ahí donde también él ha llamado, realizan lo que Freud ha aislado como la experiencia fundamental de satisfacción. Satisfacción no de la necesidad de alimentación, sino satisfacción de haber anticipado imaginariamente el objeto." (Crespin, 2004, p. 17).

Sin embargo, estas respuestas tempranas requerirán de otros tiempos en los cuales el niño a condición de ser el objeto de deseo de la madre, pierda ese lugar por la intervención del padre en tanto portador del objeto y más tarde, en tanto representante de la ley. Será en ese entretejido lógico que el síntoma advenga como respuesta ante la falta, como metáfora que dice de la historia singular de un sujeto. La posibilidad de 
separación vía la metáfora paterna sostendrá el aparecimiento del síntoma en tanto expresión fallida de la pérdida y búsqueda incesante del objeto, como diría Lacan: "el síntoma es el efecto de lo simbólico en lo real" (Chemama, 2004, p.638). Esta es la paradoja en la que se encuentra el neurótico y con la que se opera a nivel de la clínica; sin embargo y volviendo al apunte de Lacan, cuando el síntoma en el niño responde a lo sintomático de la pareja conyugal, esto implicaría que existe una posición subjetiva susceptible de intervenciones que se encaminen a la constitución de un síntoma propio en el niño, de ahí la afirmación de Lacan en cuanto a que en este caso y pese a su complejidad, habría mejores posibilidades de intervención. A este respecto Flesler nos dice: "Cuando un niño presenta síntomas a nuestra escucha, ello significa que cuenta con recursos simbólicos. Lo simbólico de la estructura está agujereado y el síntoma es solo una falla en la eficacia de la falta" (Flesler, 2007, p. 37).

No es el caso de la otra posición en la que se puede encontrar el niño y que Lacan señala como "realización" de la presencia del objeto $\boldsymbol{a}$ en el fantasma materno. Posición que implica una falla a nivel de la falta que pone en riesgo la subjetividad del niño dejándolo en la condición de un objeto que a su vez cumple la función de taponar la falta de la madre realizando su deseo. Esta circunstancia tendrá una multiplicidad de expresiones a nivel clínico, según el tiempo en el cual el niño haya quedado atrapado como objeto, en ese sentido la psicosis o las fobias podrían ser las manifestaciones más próximas a esta condición.

"Me parece que la clínica rebosa de "niños objeto del fantasma materno", en configuraciones en las cuales el padre está ausente, deficiente, ocupado en otra cosa. No necesariamente estamos en la psicosis: no es porque el niño sea el objeto del fantasma materno que él es psicótico. Pero este niño objeto de la madre colma, sutura la falta de la madre y en realidad es el caballero de la madre. No sé aquí en Ecuador, pero en Francia esos "caballeros de la madre", como yo los Ilamo, son numerosos. Y lo que es muy difícil es que ellos se aferran y se sostienen en ese lugar de falo imaginario de la madre y la madre se aferra a que el niño se quede en este lugar. Ahí los pequeños milagros de los que habla Lacan son raros, ya no se producen más estos pequeños milagros. Estamos frente a posiciones difíciles de quebrantar puesto que de ambos lados hay goce y que nosotros, psicoanalistas, vamos a atacar esas posiciones de goce. Es extremadamente difícil... si es verdad que esto sucede con los niños que tienen problemas de aprendizaje, es verdad también para los niños fóbicos, 
por supuesto que tienen angustias de separación monumentales, y es verdad también para los niños inestables que con frecuencia llegan a colmar la falta de la madre" (Bergés-Bounes, 2016, p. 20)
Dos lugares a ser tomados en cuenta al momento de escuchar el malestar en la demanda de intervención, que se explicita por parte de los padres, pero que solo en la palabra del niño encontrará su ratificación.

\section{DEMANDA Y TRANSFERENCIA}

¿Cómo llegan los niños a consulta?, esta pregunta casi siempre tendrá por respuesta el pedido de los padres o del medio educativo. No es frecuente encontrar que un niño solicite ser llevado al psicoanalista, pero cuando esto ocurre suele ser resultado de alguna intervención anterior que le permitió conocer el dispositivo.

Las entrevistas preliminares son el espacio apropiado para que el psicoanalista de lugar a que la demanda se despliegue más allá de su condición imaginaria sostenida en algún acontecimiento particular y adquiera el estatuto de deseo al cual se liga una verdad. Es también el espacio para conocer y reconocer el lugar del síntoma que la queja genera, no para ser suprimido, sino muy por el contrario, para hacer de él la causa del tratamiento. En el caso del psicoanálisis con niños, como dijimos anteriormente es el momento para ubicar ese síntoma en sus dos posibilidades de manifestación del deseo del Otro. Sin embargo, cabe preguntarse, ¿cuánto de estos planteamientos es factible de llevar a cabo al hacer clínica psicoanalítica con niños?, si tenemos en cuenta justamente que es una práctica confrontada permanentemente a la presión de lo social que exige la entrada en la "norma" como única posibilidad para ser reconocido y poder ocupar un lugar junto a los otros.

Vemos entonces que la clínica con niños exige e interroga permanentemente al deseo del analista en cuanto a su posicionamiento ético. No es sencillo reconocer al niño como un sujeto abocado a la falta, quizá esta resistencia provenga de lo que Freud denominaba: "la amnesia infantil", mecanismo por el cual atribuimos al niño una completud imaginaria que lo ubicaría en la categoría de "ángel sin tachadura" y que el social incesantemente demanda restablecer en su pedido de reeducación, lo que probablemente corresponde a la ilusión narcisística que el niño venga a ubicarse en ese lugar del ideal. Freud en 1914 ya advir- 
tió sobre ello cuando en "Introducción al narcisismo", planteaba el narcisismo primario que se supone al niño, como efecto del renacimiento y reproducción del propio narcisismo de los padres y de aquel tiempo en el cual siendo niños se les atribuyó toda clase de perfecciones y se veló y olvidó toda clase de defectos.

"Enfermedad, muerte, renuncia al goce, restricción de la voluntad propia no han de tener vigencia para el niño, las leyes de la naturaleza y de la sociedad han de cesar ante él, y realmente deber ser de nuevo el centro y el núcleo de la creación. His majesty the baby, como una vez nos creímos. Debe cumplir los sueños, los irrealizados deseos de sus padres... El conmovedor amor parental, tan infantil en el fondo, no es otra cosa que el narcisismo redivivo de los padres, que en su trasmudación al amor de objeto revela inequívoca su prístina naturaleza" (Freud, 2010, p. 88).

Deseos fundantes de todo sujeto, necesarios en los momentos iniciales de su constitución, y sin embargo a ser interrogados en otro tiempo, porque "sin tachadura" tampoco es posible el deseo y este es el campo al que apunta el psicoanálisis y la clínica con niños, campo en donde la falta, la falla, lo disarmónico, o la sorpresa tienen un lugar de escucha y en donde la oferta de curar el síntoma al responder a la demanda social estaría más bien en el terreno de la psicoterapia y no del psicoanálisis cuyo interés por el síntoma adquiere otro estatuto.

"La práctica psicoanalítica con niños debe tener en cuenta que lo que se demanda en consulta es algo que guarda relación tanto con el inconsciente de los padres como con el del niño" (Bergès, 2011, p.23). Esta pertinente observación de Bergès, nos coloca frente a varias preguntas respecto a la transferencia que se produce en el trabajo clínico con niños. ¿Es posible hablar de clínica psicoanalítica con niños, excluyendo a los padres? ¿Cuál es el lugar de los padres a nivel transferencial en el trabajo clínico con niños?

Es evidente para todo psicoanalista que trabaja con niños que la transferencia, en tanto lugar al que se le supone un saber debe establecerse a partir de los padres hacia el analista, para que un trabajo con el niño pueda sostenerse; sin embargo, Bergès ( $\mathrm{s} / \mathrm{f}$ ) plantea que en algún momento lo que empieza siendo demanda de transferencia deberá convertirse más tarde en una transferencia de la demanda.

En un inicio entonces son los padres quienes se acercan a la consulta manifestando diversos modos de hacer saber sobre su pedido de ayuda. Para Alba Flesler hay tres posicionamientos en los cuales se ubica la transferencia a nivel de los padres: cuando consultan, cuando demandan y cuando son enviados des- 
de un tercero que ha notado algún malestar que ha pasado desapercibido en el entorno familiar.

En el primer caso, cuando los padres consultan, dan cuenta de una pregunta que los convoca y suscita la búsqueda de un saber. Según Flesler, este es el posicionamiento más proclive al establecimiento simbólico de la transferencia, cuyos efectos posibilitarán el desarrollo de un trabajo propiamente psicoanalítico. Un ejemplo es el caso del padre de Juanito, el cual como conocedor de las ideas freudianas, dirige las observaciones que hace sobre el síntoma fóbico de su hijo, al doctor Freud, quien ubicado en el lugar de sujeto supuesto saber, permitirá que las preguntas al enigma que representa el síntoma, encuentren una dimensión de escucha. La transferencia que el padre de Juanito tiene con el doctor Freud será el puente que hará posible que el niño estructure sus propias preguntas y que finalmente arme sus propias teorías a las cuales pondrá a trabajar como mecanismo para instalarse en la fobia y más tarde en su resolución.

Cuando los padres demandan es el segundo posicionamiento a nivel transferencial, y que generalmente involucra a su narcisismo herido, cuando el niño no responde a sus expectativas e ideales, entonces se pide "arreglar", "curar" a ese hijo, de aquello que no funcio- na y que trae problemas al entorno. No hay una pregunta desde ellos respecto a lo que puede estar sucediendo con su hijo, muy por el contrario, el pedido se reduce a una demanda imaginaria de "cambio", donde el saber queda obturado y reducido a la sola responsabilidad del niño. En el caso Dora trabajado por Freud, Flesler ejemplifica este modo de transferencia.

"El padre de Dora no consulta, pero demanda. Desresponsabilizándose de interrogar el saber en relación a la verdad que lo implica, reclama a Freud que coloque a la joven en la buena senda, le pide que vuelva a la hija dócil, que la encuadre dándole bienestar y confort al pacto de la pareja familiar que la rebelde jovencita perturbaba con su denuncia. En su pedido funciona la transferencia pero no hay búsqueda de saber" (Flesler, 2007, p. 145)

La tercera modalidad de transferencia frecuente en la clínica con niños corresponde a cuando los padres son "enviados" por alguna instancia desde lo social, escuela, hospital, juzgado, etc., que ha registrado algún llamado a intervenir sin que los padres se sientan concernidos ni se hayan percatado de ello. Este tipo de transferencia generalmente negativa genera malestar y como lo expresa Flesler, "el analista ha de vérselas con el costado más real de la transferencia que lo enfrenta con perfiles franca- 
mente pasionales" (Flesler, 2007, p. 144). El caso de la joven homosexual es un ejemplo de este tipo de transferencia. Aquí el padre asiste sin dar mucho crédito al trabajo de Freud, no hay una suposición de saber, ni interés por el mismo, en esas condiciones se anticipa un fracaso de la intervención.

Se han abordado hasta aquí las diferentes modalidades en relación con la demanda y la transferencia de los padres en el trabajo clínico con niños, pero ¿qué pasa con la demanda y la transferencia del sujeto implicado?

En relación con esta pregunta encontramos algunas reflexiones en Bergès, quien al recoger indicaciones técnicas de Hermina von Hugh Helmut, discípula de Freud y una de las primeras psicoanalistas de niños que dan un giro en el planteamiento del trabajo clínico, hasta entonces recomendado a realizarse por intermedio de los padres, y al cual esta analista aporta nuevos elementos que se articulan con los conceptos fundamentales del psicoanálisis interrogando dicha prescripción, plantea que:

"El niño no formula al analista ninguna demanda, y va a su encuentro en contra de su voluntad, ignorando todo de las metas de una cura pero teniendo apego a su sufrimiento del cual goza para reforzar su sentimiento de omnipotencia y su narcisismo de la diferencia. Con él conviene por consecuencia crear prime- ro una relación transferencial, susceptible de permitir la emergencia de una demanda, y esta relación, para llegar a ella, se sostiene primero del narcisismo. Así es mejor que los primeros contactos con sus padres se hagan sin él y que la cura comience en su domicilio" (Bergès, s/f.).

Las formulaciones de $\mathrm{H}$. Von Hugh Helmut incluyeron la sugerencia de adaptar el método psicoanalítico a las características particulares del niño, con la finalidad que la transferencia se establezca sin interdicciones, ni sugestiones que lastimen la confianza del niño en el analista, de manera que el niño se comprometa en una transferencia positiva puramente especular que será la base de las intervenciones posteriores, encaminadas a simbolizar el lugar que ocupa en tanto objeto de deseo del Otro.

"La imago es esta moneda de intercambio de la cual el niño alimenta sus tramas de transferencia; según su signo, el analista es por turno aliado de los padres contra el niño, su aliado contra ellos, enemigo de los tres o amigo de todos; también es a veces el ideal parental o el mejor de los niños, el peor o el más solidario de una fratría... en breve, el joven analizante abandona progresivamente, gracias a la cura, sus procesos de atribución y los clivajes maniqueístas y angustiantes propias de las imágenes, para llegar a simbolizar la imago a la cual, a veces con efectos 
depresivos, puede entonces renunciar como objeto a" (Bergès, s/f).

Sin embargo, una de las críticas a la propuesta de la señora Helmut se dirige al carácter pedagógico que imprimió a sus intervenciones, por lo que se la ubica más en el campo de la psicoterapia que en el del psicoanálisis.

Conducir una cura con niños sostenida en el dispositivo de la transferen- cia implica el reconocimiento en el niño de un saber inconsciente a la espera de una escucha atenta, que acompañe al niño en la construcción de una pregunta en la que sostenga sus representaciones fantasmáticas de la realidad del deseo que lo constituye y a la que podrá retornar en lo posterior, volver a interrogar y continuar la búsqueda del saber, de la verdad y del objeto.

\section{LA CURA EN LA CLÍNICA PSICOANALÍTICA CON NIÑOS}

La noción de cura en psicoanálisis partió de la clásica idea de curar el síntoma que aqueja al sujeto, lo vemos en las primeras intervenciones de Freud con sus pacientes histéricas a las cuales deja hablar llegando a descubrir el método catártico, cuya finalidad consistía en el "desahogo" del afecto que perturbaba la vida psíquica de estas mujeres. Sin embargo a estos primeros pasos, Freud irá añadiendo nuevas reflexiones empujado por la clínica de las neurosis que lo conducen al descubrimiento del inconsciente, así como de las leyes y condiciones que lo componen. La conceptualización de la pulsión de muerte ligada a la compulsión a la repetición constituye una evidencia del permanente retorno de lo reprimido y de la función determinante del síntoma como manifestación de la estructura del sujeto. Acallar el síntoma entonces dejó de ser la finalidad de la cura en psicoanálisis, muy por el contrario el aporte freudiano establece una nueva concepción del mismo, en tanto metáfora del inconsciente, y a partir de Lacan, efecto de estructura del sujeto, en articulación con los tres registros: real, imaginario y simbólico (Chemama, 2004).

“El psicoanálisis no ataca al síntoma. El psicoanalista escucha a un niño para permitirle saber quién es. Cuando yo escucho a un niño no es para que trabaje mejor en el colegio, es para que pueda saber un poco quién es él y lo que quiere, y cuál es el lugar que tiene ganas de ocupar" (Bergés-Bounes, 2016, p. 27). El lugar que un niño ocupará en relación al deseo del Otro, es la apuesta que la clínica con niños plantea al pequeño sujeto, cuyo síntoma da cuenta de su sufrien- 
te lugar como amo del goce del Otro. Posicionamiento que le trae problemas pero al que se resiste a abandonar.

Jean Bergés (s/f) conceptualiza esta manifestación de la clínica como una no-demanda de la cual partirá el establecimiento de la transferencia con los niños y marcará la dirección de la cura, obligando al analista a ubicarse en permanente alerta frente a su propia no-demanda, única posibilidad de no caer en los pedidos ideales de lo social, de los padres y en general de la cultura que incitan a la desaparición del malestar, en defensa del "funcionamiento" colectivo.

Varios analistas consideran que la cura en la clínica con niños apunta a la constitución de un síntoma que opere en tanto función del padre, promoviendo la separación del sujeto del deseo del Otro, y dando lugar a que el niño tome posición en relación con los significantes que lo determinan y con los cuales construya un modo de ubicación frente al agujero de la castración.

"La función del síntoma es escribir el vacío de goce del no hay relación sexual por medio de la letra. El síntoma permite nombrar el vacío de goce, así como produce recuperación de goce por medio de la articulación significante propia del inconsciente. Por eso el síntoma le permite al sujeto una posición con respecto al inconsciente. Si la interpretación analítica apunta a vaciar ese goce, entonces enfrenta al sujeto con su vacío. Así la interpretación en el análisis con niños apunta a efectivizar la castración en el Otro. Así el síntoma propio del niño le permite nombrar, ubicar el vacío por eso hace operativizable la castración para él, le permite asumir una posición frente a esta. El síntoma de esta manera le posibilita al niño localizar y tener una versión del objeto 'a', le permite inscribir la pérdida de goce y operar con su propia pérdida en tanto separado del Otro, ubicándose en relación al S(A)." (Martínez \& Gross, s/f).

La cura se dirigirá entonces al reconocimiento del sujeto y de la falta que lo funda. Tener en cuenta este planteamiento permite al clínico que trabaja con niños evitar caer en la seducción de obrar buscando "el bien", del otro, expresado en la asistencia maternal, o en pretender ocupar el lugar de los padres sustituir su deseo particular por el de aquellos, o suplir sus carencias educativas.

Es la asunción del deseo inconsciente, que subyace en el síntoma a donde deben apuntar las intervenciones del analista, en ese sentido incorporar a los padres en la cura del niño, no implica trabajar simultáneamente con ellos en relación a sus propias interrogaciones subjetivas, sino reconocerlos y respetarlos en cuanto a su verdad más íntima, considerando que el niño hace parte de 
la misma y deberá encontrar en ella su origen como sujeto. En relación con esto Francoise Doltó (1987, p.185) plantea:

"Pienso ciertamente que el Edipo del niño debe hacerse con los padres; nosotros, psicoanalistas, podemos solamente ayudar al niño a superar el Edipo; pero es muy importante en su vida que no ocupemos el lugar del padre que prohíbe que la vida fantasiosa e imaginaria del niño, o incluso sus pesadillas, importunando todo lo que pasa en la casa, moleste a toda la familia. Es el padre quien ha de tomar en sí mismo la autoridad, no el psicoanalista. Cuando un niño ha estado enfermo físicamente, quedando débil o retardado, sus padres tienen mucho miedo. Pues bien, es precisamente el Edipo lo que hace cambiar la situación para el niño y para los mismos padres. Pues el niño no será un retrasado si se ayuda a los padres a aceptar el destete. ¿Destete de qué? Del hábito de llevar a su hijo al terapeuta. ¿Qué traen en realidad? Un niño en estado de transferencia. "Explíquenles esta transferencia: sean para el niño más importante que yo (médico o psicoanalista), esto es lo que les deben hacer comprender. El padre y la madre han de retomar su papel. En un adulto, la transferencia se hace con el analista; pero en un niño, el Edipo se hace con los padres; no es transferible al analista"

Finalmente es necesario anotar como uno de los puntos más importan- tes en la dirección de la cura en la clínica con niños, el sostenimiento de las intervenciones del analista desde el plano simbólico, es decir operando como terceridad que viene a reorganizar el goce al que el niño se ha entregado con su síntoma. Lugar de alteridad que el analista encarna en tanto representación del Otro y en donde el sujeto encontrará el límite que lo estructure en tanto tal.

"Es verdad que el psicoanálisis con los niños nos coloca, a veces, frente a escenas; es decir que no se trata como con un adulto que habla, pero lo vemos. Algunas veces encuentro a niños que están con sus dos manos en los senos de su mamá, la cartera de la mamá es vaciada, y de tiempo en tiempo me pregunto qué puedo hacer para detener este espectáculo, que a mí me resulta insoportable, y me digo, ¿en qué lugar está este niño, que él tenga otra posición?, y son cosas que nos dan para que sean vistas, y somos cómplices si lo miramos, porque en ese momento por supuesto van a añadir más. Entonces, cómo no ser cómplice y detener un goce que solo nos importa a nosotros, y finalmente, en nombre de qué, una vez más, en nombre de qué vamos a detener este goce" (Bérges-Bounes, 2016, p. 121).

Preguntas que apuntan a lo simbólico de la transferencia, como posibilidad de intervención que haga corte y cuyo efecto produzca la restricción de 
un goce cuyo acceso a una condición fálica se encuentra obturado.

Es esta la especificidad de la clínica psicoanalítica con niños que se di- rige en torsión de banda de moebius al sujeto del lenguaje ubicando su lugar en tanto objeto del deseo del Otro y a los efectos que de ello deriva.

\section{CONCLUSIONES}

La clínica psicoanalítica con niños es un campo cuya especificidad ha sido abordada desde diversas conceptualizaciones del sujeto al que apunta esta práctica. Sin embargo, muchas de ellas han dado lugar a deslizamientos que han conducido el trabajo con los niños hacia la intervención psicoterapéutica, olvidando el fundamento epistemológico del psicoanálisis cuyo objeto de estudio es el sujeto del inconsciente.

Un retorno a los fundamentos de la práctica psicoanalítica y a aportes como la lectura que hace Lacan del sujeto freudiano, ubicándolo como efecto del lenguaje, son ejes en los que la clínica psicoanalítica con niños encuentra la rigurosidad de una práctica fácilmente derivada al cumplimiento del encargo social, que espera de su accionar el "funcionamiento" del niño en tanto ideal en el que se deposita el devenir de la cultura.

Una clínica psicoanalítica con niños que apunta al sujeto y sus modos de manifestación en los entretejidos del lenguaje con los que el síntoma se hace escuchar, implica un reconocimiento del doble movimiento en el que se encuentra el infans, en tanto objeto del deseo del Otro. De ahí la importancia de un trabajo clínico que, otorgando un lugar a la palabra de los padres, sin embargo no pierda de vista la apuesta por el sujeto.

La ética del psicoanálisis es una ética del sujeto del inconsciente, es decir de aquel que nace del reencuentro con el universo del lenguaje y en el traumatismo de la pérdida del objeto, de ahí que la clínica psicoanalítica con niños se dirige a desentrañar el lugar del deseo en el pequeño sujeto entretejido en el deseo de sus padres, por lo que el discernimiento de la demanda en las entrevistas preliminares permitirán ir delineando la posibilidad de la transferencia con el niño. Serán los lapsus, los actos fallidos, los sueños, las claves para conocer el deseo de iniciar un trabajo psicoanalítico, más allá de la respuesta afirmativa que un niño pueda emitir al ser convocado a trabajar.

En "Nota sobre el Niño", Lacan dará cuenta del lugar que el síntoma tiene en 
el niño, abriendo el camino para la dirección de la cura en el reconocimiento del deseo como no anónimo, planteamiento a considerar en todo momento cuando se interviene con un niño y que introduce al clínico en la interrogación constante sobre su lugar en la transferencia, la misma que se juega en esa doble consideración del deseo, donde el lugar de la alteridad apuntalada en lo simbólico de la transferencia, marcará la posibilidad de intervenir cortando el goce del cual el niño es su amo.
Finalmente quedan varias interrogaciones que suscitan la continuidad de esta reflexión; sin embargo, es importante enunciar una de ellas por su pertinencia en relación con el tema del presente ensayo: ¿si la especificidad de la clínica psicoanalítica con niños, implica sostener en la transferencia la construcción del síntoma en el niño, entonces qué otras consideraciones se deben tener en cuenta al posicionarse como analista y operar desde el lugar de semblante de objeto? 


\section{BIBLIOGRAFIA}

Bergès, J. (2011). Le corps de l'enfant, corps parlé, imaginé ou stigmatisé. Toulouse: Érès.

Bèrges, J. (s/f). El encuadre de la cura: demanda, transferencia y contrato con los padres y para su hijo. Recuperado de: www.freud-lacan. com/.../347-El_encuadre_de_la_ cura_demanda

Bergès, M. (2016). Clínica psicoanalítica con niños anudamiento cuerpo-lenguaje. Grupo a..b..c...dario Freud-Lacan. Quito: Rimana

Chemama, R. (2004). Diccionario del Psicoanálisis. Buenos Aires: Amorrortu.

Crespin, G. (2004). Aspects cliniques et pratiques de la prevention de l'autisme. Paris: L'Harmattan.

Doltó, F. (1987). Seminario de psicoanálisis con niños. México: Siglo XXI.

Flesler, A. (2007). El niño en análisis y el lugar de los padres. Buenos Aires: Paidós.

Freud, S. (2010). Introducción al Narcisismo (1914). Obras Completas. Tomo XIV. Buenos Aires: Amorrortu.

Klein, M. (1989). Essais de Psychanalyse (1968). Paris: Payot.

Lacan, J. (2012). Otros Escritos. Buenos Aires: Paidós.

Martinez L. \& Gross G. (s/f). El niño y el sintoma. Recuperado de: http:// www.fort-da.org/fort-da2/sintoma.htm)

Rey C. \& Bergés-Bounes M. (2008). L'enfant et le symbolique en Sex and Gender. Le bulletin lacanien. No 4. Páginas: 90-94. París: ALI. 